\title{
CHARACTERIZATION OF BALLS BY RIESZ-POTENTIALS
}

\author{
WOLFGANG REICHEL
}

\begin{abstract}
For a bounded convex domain $G \subset \mathbb{R}^{N}$ and $2<\alpha \neq N$ consider the unitdensity Riesz-potential $u(x)=\int_{G}|x-y|^{\alpha-N} d y$. We show in this paper that $u=$ const. on $\partial G$ if and only if $G$ is a ball. This result corresponds to a theorem of L.E. Fraenkel, where the ball is characterized by the Newtonian-potential $(\alpha=2)$ of unit density being constant on $\partial G$. In the case $\alpha=N$ the kernel $|x-y|^{\alpha-N}$ is replaced by $-\log |x-y|$ and a similar characterization of balls is given. The proof relies on a recent variant of the moving plane method which is suitable for Green-function representations of solutions of (pseudo-)differential equations of higher-order.
\end{abstract}

\section{INTRODUCTION}

In Newton's theory of gravitation the potential of a ball $B_{R}(0) \subset \mathbb{R}^{3}$ of constant mass density $\rho>0$ is given by

$$
u(x)=\frac{1}{4 \pi} \int_{B_{R}(0)} \frac{\rho}{|x-y|} d y= \begin{cases}\rho\left(\frac{R^{2}}{2}-\frac{|x|^{2}}{6}\right), & |x| \leq R, \\ \frac{\rho R^{3}}{3|x|}, & |x| \geq R .\end{cases}
$$

Outside the ball the gravitational potential coincides with that of a single point centered at the origin whose mass equals the mass of the entire ball. This observation (and its generalization to radially symmetric mass densities) allows to reduce celestial mechanics of stars and planets to the interaction of point masses. Similar properties hold for the Newtonian potential of an $N$-dimensional ball $N \geq 4$ and for the two-dimensional logarithmic potential of a disk in $\mathbb{R}^{2}$. Note that the gravitational potential of a ball of constant mass density is constant on the surface of the ball. This property in fact uniquely characterizes the balls, as it was shown by Fraenkel [7] through the following theorem.

Theorem 1 (Fraenkel, 2000). Let $G \subset \mathbb{R}^{N}$ be a bounded open set and let $\omega_{N}$ be the surface measure of the unit-sphere in $\mathbb{R}^{N}$. Consider

$$
u(x)= \begin{cases}\frac{1}{2 \pi} \int_{G} \log \frac{1}{|x-y|} d y, & N=2, \\ \frac{1}{(N-2) \omega_{N}} \int_{G} \frac{1}{|x-y|^{N-2}} d y, & N \geq 3 .\end{cases}
$$

If $u$ is constant on $\partial G$ then $G$ is a ball.

One of the striking aspects of Fraenkel's theorem is that no regularity of $G$ is assumed a-priori. The goal of this paper is to prove for Riesz-potentials the following analogue of the above result. Unlike in Theorem 1 we need to a-priori restrict the class of open sets.

Date: October 24, 2007.

2000 Mathematics Subject Classification. Primary: 31B30, 35J30; Secondary: 31B35, 35S99.

Key words and phrases. Riesz-potential, pseudo-differential operator, moving plane method, radial symmetry. 
Theorem 2. Let $G \subset \mathbb{R}^{N}$ be a bounded convex domain. For $\alpha>2$ consider

$$
u(x)= \begin{cases}\int_{G} \log \frac{1}{|x-y|} d y, & N=\alpha, \\ \int_{G} \frac{1}{|x-y|^{N-\alpha}} d y, & N \neq \alpha .\end{cases}
$$

If $u$ is constant on $\partial G$ then $G$ is a ball.

It is easy to see that the converse of both Theorem 1 and Theorem 2 hold. Suppose $G=B_{R}(0)$ is a ball centered at the origin. Then $u$ is radially symmetric and hence $u$ is constant on $\partial G$.

Let us give some heuristic arguments for Fraenkel's theorem. The Newtonian potential in Theorem 1 satisfies

$$
-\Delta u=1 \text { in } G, \quad-\Delta u=0 \text { in } \mathbb{R}^{N} \backslash \bar{G}
$$

and by assumption $u=\beta$ on $\partial G$. If one considers the two boundary value problems (here we assume $N \geq 3$ )

$$
(*)\left\{\begin{array} { l } 
{ - \Delta u _ { i } = 1 \text { in } G , } \\
{ u _ { i } = \beta \text { on } \partial G }
\end{array} ( * * ) \left\{\begin{array}{l}
-\Delta u_{e}=0 \text { in } \mathbb{R}^{N} \backslash \bar{G} \\
u_{e}=\beta \text { on } \partial G, u_{e} \rightarrow 0 \text { at } \infty
\end{array}\right.\right.
$$

then there exist unique solutions $u_{i}, u_{e}$, and they must coincide with $u$. The fact that $u$ is a $C^{1}\left(\mathbb{R}^{N}\right)$ function means that next to the boundary values $u_{i}=u_{e}=\beta$ on $\partial G$ also the normal derivatives of $u_{i}, u_{e}$ have to coincide on $\partial G$. For an arbitrary domain $G$ this would not be the case. Thus, $(*),(* *)$ together with matched normal derivatives is an overdetermined problem, which explains why the shape of $G$ cannot be arbitrary. Infact, the only way to resolve $(*),(* *)$ and simultaneously match the normal derivatives is by $G$ being a ball. Note that in Fraenkel's theorem no regularity of $\partial G$ is assumed, so that in general normal derivatives of $u_{i}, u_{e}$ cannot be understood in the classical sense.

Let us discuss similarly the Riesz-potentials of Theorem 2. First we recall fundamental solutions $G(x, y)$ of the pseudo-differential operators $(-\Delta)^{\alpha / 2}$ in $\mathbb{R}^{N}, \alpha>0$. In case $\frac{1}{2}(\alpha-N) \notin \mathbb{N}_{0}$ (i.e., either $0<\alpha<N$ or $\alpha \geq N$ but $\alpha-N$ is not an even natural number) then

$$
G(x, y)=\frac{\Gamma\left(\frac{N-\alpha}{2}\right)}{2^{\alpha} \pi^{N / 2} \Gamma\left(\frac{\alpha}{2}\right)}|x-y|^{\alpha-N}
$$

whereas if $\alpha-N=2 k, k \in \mathbb{N}_{0}$ then

$$
G(x, y)=\frac{(-1)^{k}}{2^{\alpha-1} \pi^{N / 2} \Gamma\left(\frac{\alpha}{2}\right)}|x-y|^{\alpha-N} \log \frac{1}{|x-y|} .
$$

It follows that for $(\alpha-N) / 2 \notin \mathbb{N}_{0}$ the potential $u$ of Theorem 2 satisfies in the distributional sense $\left(\chi_{G}\right.$ is the characteristic function of the set $\left.G\right)$

$$
(-\Delta)^{\alpha / 2} u=\text { const. } \chi_{G} \text { in } \mathbb{R}^{N}
$$

together with $u=\beta$ on $\partial G, u \in C^{l}\left(\mathbb{R}^{N}\right)$ for $1 \leq l<\alpha$. Note that for $\alpha=2 m$ the potential $u$ satisfies a polyharmonic equation in $\mathbb{R}^{N}$. For general $\alpha>2$ there is no analogue of the two boundary value problems $(*),(* *)$ as in the second-order case. It is therefore remarkable that the mere information of $G$ being a level set of $u$ completely determines $u$ and $G$. Even in the case $\alpha=2 m$ the boundary value problems analogous to $(*),(* *)$ are underdetermined individually since only one boundary datum is prescribed. 
But if they are viewed as a system coupled by the fact that $u \in C^{2 m-1}\left(\mathbb{R}^{N}\right)$ coincides with $u_{i}$ in $G$ and $u_{e}$ outside $G$ then they become overdetermined.

We finish this discussion with the following two open problems:

(i) Is Theorem 2 true if the assumption of convexity of $G$ is dropped?

(ii) Is there an analogous result as in Theorem 2 for potentials

$$
u(x)=\int_{G}|x-y|^{\alpha-N} \log \frac{1}{|x-y|} d y ?
$$

The most interesting case would be the case where $\alpha-N=2 k$ with $k \in \mathbb{N}_{0}$ since then the kernel function is (up to a normalization constant) the fundamental solution of $(-\Delta)^{\alpha / 2}$.

The main reason why both questions remain open is the fact that the validity of Lemma 4 is not clear under these assumptions, cf. the remark following Lemma 4.

A number of potential-theoretic characterizations of balls are known in the literature. If instead of a volume potential one considers a single-layer potential $u$ concentrated on $\partial G$ with constant density, then $G$ is a ball if and only if $u$ is constant on $\partial G$. This conjecture of P. Gruber (cf. Heil, Martini [10]) has been verified for different smoothness classes of domains. The two-dimensional case was considered by Martensen [13], Gardiner [8] and Ebenfelt et. al. [6] and the higher-dimensional case by Reichel [17], Mendez and Reichel [14] and Sirakov [19]. We mention that in [14] only convexity of the underlying domain was assumed. Similar characterizations of annuli were given by Payne, Philippin [15] and Philippin [16] and different single-layer characterizations of balls were achieved by Shahgholian [20] and Mikyoung Lim [12].

Our approach is based on a new variant of the moving plane method. The classical moving plane method is based on the pointwise maximum principle for second order elliptic equations. It was developed by Alexandrov [1], Serrin [18] and Gidas, Ni, Nirenberg [9]. Very recently some important improvements of the moving plane method were achieved by Chang, Yang [4], Berchio, Gazzola, Weth [2], Li [11], Chen, Li, Ou [5] and Birkner, López-Mimbela, Wakolbinger [3]. These new variants of the moving plane method are applied to the integral equation resulting from the Green-function representation, cf. Lemma 10 below. In this way symmetry results for higher-order elliptic problems as well as pseudo-differential equations can be achieved although pointwise maximum principles are not available.

The paper is organized as follows. In Section 2 we provide some basic estimates for the far-field of the potential. In Section 3 the moving-plane procedure is carried out.

\section{Estimates for the Riesz-Potentials}

Throughout the paper let $\alpha>2$ and let $u$ denote the function defined in (1).

Lemma 3. Let $l \in \mathbb{N}$ with $1 \leq l<\alpha$. Then $u \in C^{l}\left(\mathbb{R}^{N}\right)$ and differentiation of order $l$ can be taken under the integral.

Proof. The result is standard. We give a proof for the reader's convenience. We consider the case $\alpha \neq N$; the proof for $\alpha=N$ is just a slight variant. Let $\eta:[0, \infty) \rightarrow[0,1]$ be a $C^{\infty}$-function with $\eta \equiv 0$ on $[0,1]$ and $\eta \equiv 1$ on $[2, \infty)$. Let $\mu=\left(\mu_{1}, \ldots, \mu_{N}\right)$ be a multi-index of order $|\mu|=l$ and let $c_{1}(l), c_{2}(l), \ldots$ denote constants which only depend 
on $l$. For $\epsilon>0$ let $\eta_{\epsilon}(t):=\eta(t / \epsilon)$ and define

$$
u_{\epsilon}(x):=\int_{G} \frac{\eta_{\epsilon}(|x-y|)}{|x-y|^{N-\alpha}} d y, \quad v_{\mu}(x):=\int_{G} D_{x}^{\mu} \frac{1}{|x-y|^{N-\alpha}} d y .
$$

Note that $\left|D_{x}^{\mu}\right| x-\left.y\right|^{\alpha-N} \mid \leq$ const. $|x-y|^{\alpha-N-l}$ with $\alpha-N-l>-N$. Therefore $v_{\mu}(x)$ exists for all $x \in \mathbb{R}^{N}$. Furthermore

$$
\begin{aligned}
\left|D^{\mu} u_{\epsilon}(x)-v_{\mu}(x)\right| & \leq \int_{G} D_{x}^{\mu}\left(\left(1-\eta_{\epsilon}(|x-y|)\right)|x-y|^{\alpha-N}\right) d y \\
& \leq c_{1}(l) \sum_{|\nu|+\left|\nu^{\prime}\right|=l} \int_{G} D_{x}^{\nu}\left(1-\eta_{\epsilon}(|x-y|)\right) D_{x}^{\nu^{\prime}}|x-y|^{\alpha-N} d y \\
& \leq c_{2}(l) \sum_{|\nu|+\left|\nu^{\prime}\right|=l} \int_{G} \epsilon^{-|\nu|}|x-y|^{\alpha-N-\left|\nu^{\prime}\right|} d y \\
& \leq c_{3}(l) \epsilon^{\alpha-l} \rightarrow 0 \text { as } \epsilon \rightarrow 0 .
\end{aligned}
$$

Thus $D^{\mu} u_{\epsilon}$ converges uniformly on $\mathbb{R}^{N}$ to $v_{\mu}$ for all multi-indices $\mu$ with $|\mu|<\alpha$. This establishes the proof.

In the following we assume that $G$ is convex and that $u=$ const. $=\beta$ on $\partial G$.

Lemma 4. If $N \geq \alpha$ then $u(x)<\beta$ for $x \in \mathbb{R}^{N} \backslash \bar{G}$ and $u(x)>\beta$ for $x \in G$. If $N<\alpha$ then $u(x)>\beta$ for $x \in \mathbb{R}^{N} \backslash \bar{G}$ and $u(x)<\beta$ for $x \in G$.

Remark. In the computations below we use that the kernel function $|x-y|^{\alpha-N}$ has monotonicity and sub-/superharmonicity properties. In general this is not the case for kernels of the form $|x-y|^{\alpha-N} \log 1 /|x-y|$. Moreover, it is an open problem how to overcome the convexity assumption of $G$ in the proof below.

Proof. Lemma 3 shows that $u$ is a $C^{2}\left(\mathbb{R}^{N}\right)$-function since $\alpha>2$. Note that $\Delta|x|^{\alpha-N}=$ $(\alpha-N)(\alpha-2)|x|^{\alpha-N-2}$ and $\Delta \log \frac{1}{|x|}=(2-N)|x|^{-2}$. Let us first consider the case $N \geq \alpha>2$. In this case $u$ is superharmonic and hence inside $G$ the function $u$ is larger than the value $\beta$ of $u$ on $\partial G$. In the case $2 \leq N<\alpha$ the function $u$ is subharmonic and hence inside $G$ the function $u$ is smaller than its value $\beta$ on the boundary. It remains to consider $u$ outside $G$. We show that the convexity of $G$ implies that $u$ has no local extremum outside $G$. Since either $u(x) \rightarrow 0, \infty$ or $-\infty$ as $|x| \rightarrow \infty$ this implies that $u$ is smaller (larger) than $\beta$ outside $G$. So let $x \in \mathbb{R}^{N} \backslash \bar{G}$. By the convexity of $G$ we can separate $x$ from $G$ through a hyperplane, i.e., there exists a unit vector $e \in \mathbb{R}^{N}$ and a point $z_{0} \in \mathbb{R}^{N} \backslash \bar{G}$ such that

$$
\left(y-z_{0}\right) \cdot e<0<\left(x-z_{0}\right) \cdot e \text { for all } y \in G .
$$

In particular $(x-y) \cdot e>0$ for all $y \in G$. Since

$$
\nabla u(x) \cdot e=c_{\alpha, N} \int_{G} \frac{(x-y) \cdot e}{|x-y|^{N-\alpha+1}} d y
$$

and the integrand is strictly positive we see that $u$ has no local extremum outside $G$.

By Lemma 4 we see that $G$ is a sub- or super-level set of $u$. This observation led Fraenkel [7] to rewrite $u$ as the Newtonian potential of the nonlinear density function $f_{H}(u(x)-\beta)$ over all of $\mathbb{R}^{N}$, where $f_{H}$ is the Heaviside-function. Hence $u$ fulfilled a nonlinear integral equation in $\mathbb{R}^{N}$ with no explicit appearance of the set $G$. The same is clearly true in the context of Riesz-potentials as expressed by the following corollary. 
Corollary 5. Let $f_{H}(t)=1$ for $t>0$ and $f_{H}(t)=0$ for $t \leq 0$ be the Heavisidefunction and $\chi_{G}$ be the characteristic function of $G$. Then $\chi_{G}=f_{H}(u-\beta)$ if $N \geq \alpha$ and $\chi_{G}=f_{H}(\beta-u)$ if $N<\alpha$. Hence

$$
u(x)= \begin{cases}\int_{\mathbb{R}^{N}} \log \frac{1}{|x-y|} f_{H}(u(y)-\beta) d y, & N=\alpha, \\ \int_{\mathbb{R}^{N}} \frac{f_{H}(u(y)-\beta)}{|x-y|^{N-\alpha}} d y, & N>\alpha, \\ \int_{\mathbb{R}^{N}} \frac{f_{H}(\beta-u(y))}{|x-y|^{N-\alpha}} d y, & N<\alpha .\end{cases}
$$

Lemma 6. Let $q=\frac{1}{\operatorname{vol} G} \int_{G} y d y$ be the barycentre of $G$ and let $v(x)=u(x+q)$. Then

$$
v(x)=\left\{\begin{array}{cc}
\operatorname{vol} G \log \frac{1}{|x|}+h(x) & \text { if } N=\alpha, \\
\operatorname{vol} G|x|^{\alpha-N}+h(x) & \text { if } N \neq \alpha
\end{array}\right.
$$

and there exists a constant $C>0$ such that $|h(x)| \leq C|x|^{\alpha-N-2},|\nabla h(x)| \leq C|x|^{\alpha-N-3}$.

Proof. Let $N \neq \alpha$. A direct application of Taylor's theorem to the function $g(t):=$ $|x-t \eta|^{\alpha-N}$ yields

$$
|x-\eta|^{\alpha-N}=|x|^{\alpha-N}-(\alpha-N)|x|^{\alpha-N-2} x \cdot \eta+k(x, \eta)
$$

where there exists a constant $C>0$ and a radius $R_{0}>0$ such that

$$
|k(x, \eta)| \leq C|x|^{\alpha-N-2}, \quad\left|\nabla_{x} k(x, \eta)\right| \leq C|x|^{\alpha-N-3} \quad \text { for all }|x| \geq R_{0}, \eta \in G-q .
$$

Here $R_{0}>0$ is chosen such that $\bar{G}-q \subset B_{R_{0}}(0)$. Note that

$$
v(x)=\int_{G} \frac{1}{|x+q-y|^{N-\alpha}} d y=\int_{G-q} \frac{1}{|x-\eta|^{N-\alpha}} d \eta
$$

so that the claim of the lemma follows from (2), (3) and the fact that the barycentre of $G-q$ is zero. The proof for $N=\alpha$ is similar.

\section{Proof of Theorem 2 By the method of moving planes}

For a point $x \in \mathbb{R}^{N}$ let $x^{\lambda}=\left(2 \lambda-x_{1}, x^{\prime}\right)$ be the reflection of $x$ at the hyperplane $T_{\lambda}:=\left\{x \in \mathbb{R}^{N}: x_{1}=\lambda\right\}$. Hence $\left|x^{\lambda}\right|^{2}-|x|^{2}=4 \lambda\left(\lambda-x_{1}\right)$. Also define the halfspace $H_{\lambda}:=\left\{x \in \mathbb{R}^{N}: x_{1}<\lambda\right\}$ and note that $\partial H_{\lambda}=T_{\lambda}$. On $\bar{H}_{\lambda}$ define the function $w_{\lambda}(x):=v(x)-v\left(x^{\lambda}\right)$. We will show that for $\alpha \leq N(\alpha>N)$ the function $w_{\lambda}$ satisfies

$$
w_{\lambda}(x)>0(<0) \text { in } H_{\lambda}, \quad \frac{\partial w_{\lambda}}{\partial x_{1}}(x)=2 \frac{\partial v}{\partial x_{1}}(x)<0(>0) \text { on } T_{\lambda}
$$

for all $\lambda>0$. By continuity this implies for $\alpha \leq N$ that $v\left(x_{1}, x^{\prime}\right) \geq v\left(-x_{1}, x^{\prime}\right)$ for all $x \in \mathbb{R}^{N}, x_{1} \geq 0$ while for $\alpha>N$ the reverse inequality holds. In both cases the corresponding reverse inequalities also hold by repeating the moving plane argument with the $-x_{1}$-direction. Hence $v\left(-x_{1}, x^{\prime}\right)=v\left(x_{1}, x^{\prime}\right)$ for all $x \in \mathbb{R}^{N}$ and moreover $v$ is strictly monotone in the positive $x_{1}$-direction. Repeating the moving-plane argument with an arbitrary unit-direction instead of the $x_{1}$-direction one obtains that the function $v$ is radially symmetric with respect to the origin and moreover radially strictly monotone. Together with the fact that $\partial(G-q)$ is a level-surface of the function $v$ this implies that $G-q$ must be a ball centered at the origin. Thus, Theorem 2 is proved if we show (4) for all values of $\lambda>0$. This will be done next. Theorem 2 follows from the preceeding explanation and Lemma 10 and Lemma 12. 
Lemma 7. For every $\lambda>0$ there exists a value $R(\lambda)>0$ such that for all $x \in H_{\lambda}$ with $|x| \geq R(\lambda)$ we have

$$
w_{\lambda}(x) \begin{cases}>0 & \text { if } 2<\alpha \leq N \\ <0 & \text { if } \alpha>N .\end{cases}
$$

The function $R(\lambda)$ and a value $\lambda_{0}>0$ can be chosen such that $R(\lambda)$ is non-increasing in $\lambda$ and constant for $\lambda \geq \lambda_{0}>0$.

Proof. According to the value of $\alpha$ we divide the proof into several cases. If $h$ is the function of Lemma 6 then

$$
v(x)-v\left(x^{\lambda}\right)= \begin{cases}\operatorname{vol} G\left(|x|^{\alpha-N}-\left|x^{\lambda}\right|^{\alpha-N}\right)+h(x)-h\left(x^{\lambda}\right), & \alpha \neq N \\ \operatorname{vol} G\left(-\log |x|+\log \left|x^{\lambda}\right|\right)+h(x)-h\left(x^{\lambda}\right), & \alpha=N .\end{cases}
$$

Case 1: $2<\alpha<N$. Assume first that $\left|x^{\lambda}\right|^{2} \leq 2|x|^{2}$. By convexity of the function $s \mapsto s^{\frac{\alpha-N}{2}}$ for $s>0$ we have

$$
|x|^{\alpha-N}-\left|x^{\lambda}\right|^{\alpha-N}>\frac{N-\alpha}{2}\left|x^{\lambda}\right|^{\alpha-N-2} 4 \lambda\left(\lambda-x_{1}\right) \geq C_{1}|x|^{\alpha-N-2} \lambda\left(\lambda-x_{1}\right)
$$

where $C_{1}:=(N-\alpha) 2^{\frac{\alpha-N}{2}}$. By Lemma $6\left|h(x)-h\left(x^{\lambda}\right)\right| \leq 2 C|x|^{\alpha-N-3}\left(\lambda-x_{1}\right)$. Hence

$$
v(x)-v\left(x^{\lambda}\right)>|x|^{\alpha-N-3}\left(\lambda-x_{1}\right)\left(\operatorname{vol} G C_{1}|x| \lambda-2 C\right)>0
$$

provided $|x|>\frac{2 C}{\operatorname{vol} G C_{1} \lambda}$. Next assume that $\left|x^{\lambda}\right|^{2} \geq 2|x|^{2}$. Then

$$
|x|^{\alpha-N}-\left|x^{\lambda}\right|^{\alpha-N} \geq|x|^{\alpha-N}\left(1-2^{\frac{\alpha-N}{2}}\right)=: C_{2}|x|^{\alpha-N},
$$

where $C_{2}>0$. Again by Lemma $6\left|h(x)-h\left(x^{\lambda}\right)\right| \leq 2 C|x|^{\alpha-N-2}$. Thus

$$
v(x)-v\left(x^{\lambda}\right) \geq|x|^{\alpha-N}\left(\operatorname{vol} G C_{2}-\frac{2 C}{|x|^{2}}\right)>0
$$

provided $|x|>\sqrt{\frac{2 C}{\operatorname{vol} G C_{2}}}$. Hence the statement of the lemma follows if we set

$$
R(\lambda):=\max \left\{\frac{2 C}{\operatorname{vol} G C_{1} \lambda}, \sqrt{\frac{2 C}{\operatorname{vol} G C_{2}}}\right\} .
$$

Case 2: $\alpha=N$. The structure of proof is the same as in Case 1. Assume first that $\left|x^{\lambda}\right|^{2} \leq 2|x|^{2}$. The convexity of the function $s \mapsto-\log s$ for $s>0$ implies

$$
-\log |x|+\log \left|x^{\lambda}\right|>\left|x^{\lambda}\right|^{-2} 2 \lambda\left(\lambda-x_{1}\right) \geq \frac{1}{2}|x|^{-2} \lambda\left(\lambda-x_{1}\right) .
$$

With the estimate for $h$ as above we find $v(x)-v\left(x^{\lambda}\right)>0$ provided $|x|>\frac{4 C}{\operatorname{vol} G \lambda}$. Likewise, if $\left|x^{\lambda}\right|^{2} \geq 2|x|^{2}$ then

$$
-\log |x|+\log \left|x^{\lambda}\right| \geq \frac{1}{2} \log 2
$$

and with the estimate for $h$ as above we find $v(x)-v\left(x^{\lambda}\right)>0$ provided $|x|>\sqrt{\frac{4 C}{\operatorname{vol} G \log 2}}$. Hence we may set

$$
R(\lambda):=\max \left\{\frac{4 C}{\operatorname{vol} G \lambda}, \sqrt{\frac{4 C}{\operatorname{vol} G \log 2}}\right\} .
$$

Case 3: $N<\alpha<N+2$. Again we assume first that $\left|x^{\lambda}\right|^{2} \leq 2|x|^{2}$. The concavity of the function $s \mapsto s^{\frac{\alpha-N}{2}}$ for $s>0$ implies

$$
|x|^{\alpha-N}-\left|x^{\lambda}\right|^{\alpha-N}<\frac{N-\alpha}{2}\left|x^{\lambda}\right|^{\alpha-N-2} 4 \lambda\left(\lambda-x_{1}\right) \leq-C_{1}|x|^{\alpha-N-2} \lambda\left(\lambda-x_{1}\right)
$$


with $C_{1}:=(\alpha-N) 2^{\frac{\alpha-N}{2}}$. Using the estimate for $h$ as in Case 1 we find

$$
v(x)-v\left(x^{\lambda}\right)<|x|^{\alpha-N-3}\left(\lambda-x_{1}\right)\left(-\operatorname{vol} G C_{1}|x| \lambda+2 C\right)<0
$$

provided $|x|>\frac{2 C}{\operatorname{vol} G C_{1} \lambda}$. For $\left|x^{\lambda}\right|^{2} \geq 2|x|^{2}$ we get

$$
|x|^{\alpha-N}-\left|x^{\lambda}\right|^{\alpha-N} \leq|x|^{\alpha-N}\left(1-2^{\frac{\alpha-N}{2}}\right)=:-C_{2}|x|^{\alpha-N}
$$

where $C_{2}>0$. Together with the estimate $\left|h(x)-h\left(x^{\lambda}\right)\right| \leq 2 C|x|^{\alpha-N-2}$ we obtain

$$
v(x)-v\left(x^{\lambda}\right)<|x|^{\alpha-N}\left(-\operatorname{vol} G C_{2}+\frac{2 C}{|x|^{2}}\right)<0
$$

provided $|x|>\sqrt{\frac{2 C}{\operatorname{vol} G C_{2}}}$. Therefore it suffices to set

$$
R(\lambda):=\max \left\{\frac{2 C}{\operatorname{vol} G C_{1} \lambda}, \sqrt{\frac{2 C}{\operatorname{vol} G C_{2}}}\right\} .
$$

Case 4: $\alpha \geq N+2$. For $\left|x^{\lambda}\right|^{2} \leq 2|x|^{2}$ the convexity of $s \mapsto s^{\frac{\alpha-N}{2}}$ for $s>0$ implies

$$
|x|^{\alpha-N}-\left|x^{\lambda}\right|^{\alpha-N}<\frac{N-\alpha}{2}|x|^{\alpha-N-2} 4 \lambda\left(\lambda-x_{1}\right)=:-C_{1}|x|^{\alpha-N-2} \lambda\left(\lambda-x_{1}\right)
$$

where $C_{1}=2(\alpha-N)>0$. For $h$ we obtain this time a different estimate:

$$
\begin{aligned}
\left|h(x)-h\left(x^{\lambda}\right)\right| & \leq \begin{cases}2 C\left|x^{\lambda}\right|^{\alpha-N-3}\left(\lambda-x_{1}\right) & \text { if } \alpha-N-3 \geq 0, \\
2 C|x|^{\alpha-N-3}\left(\lambda-x_{1}\right) & \text { if } \alpha-N-3<0\end{cases} \\
& \leq D|x|^{\alpha-N-3}\left(\lambda-x_{1}\right),
\end{aligned}
$$

where either $D=2^{\frac{\alpha-N-1}{2}} C$ or $D=2 C$. Thus

$$
v(x)-v\left(x^{\lambda}\right)<|x|^{\alpha-N-3}\left(\lambda-x_{1}\right)\left(-\operatorname{vol} G C_{1}|x| \lambda+D\right)<0
$$

provided $|x|>\frac{D}{\operatorname{vol} G C_{1} \lambda}$. Finally, if $\left|x^{\lambda}\right|^{2} \geq 2|x|^{2}$ then

$$
|x|^{\alpha-N}-\left|x^{\lambda}\right|^{\alpha-N} \leq\left|x^{\lambda}\right|^{\alpha-N}\left(2^{\frac{N-\alpha}{2}}-1\right)=:-C_{2}\left|x^{\lambda}\right|^{\alpha-N}
$$

where $C_{2}>0$. Together with the estimate $\left|h(x)-h\left(x^{\lambda}\right)\right| \leq 2 C\left|x^{\lambda}\right|^{\alpha-N-2}$ we conclude

$$
v(x)-v\left(x^{\lambda}\right)<\left|x^{\lambda}\right|^{\alpha-N}\left(-\operatorname{vol} G C_{2}+\frac{2 C}{\left|x^{\lambda}\right|^{2}}\right)<0
$$

provided $|x|>\sqrt{\frac{2 C}{\operatorname{vol} G C_{2}}}\left(\right.$ recall that $\left|x^{\lambda}\right| \geq|x|$ in $\left.H_{\lambda}\right)$. Therefore let us set in this case

$$
R(\lambda):=\max \left\{\frac{D}{\operatorname{vol} G C_{1} \lambda}, \sqrt{\frac{2 C}{\operatorname{vol} G C_{2}}}\right\} .
$$

Lemma 8. There exists $\lambda^{*}>0$ such that for all $\lambda>\lambda^{*}$ we have

$$
w_{\lambda}(x) \begin{cases}>0 & \text { if } 2<\alpha \leq N, \\ <0 & \text { if } \alpha>N .\end{cases}
$$

in $H_{\lambda}$. 
Proof. The proof is again divided according to the value of $\alpha$. Let $R(\lambda)$ be the function defined in Lemma 7.

Case 1: $2<\alpha<N$. Let $c_{1}:=\min _{|x| \leq R(1)} v(x)$. Hence $c_{1}>0$, and since $v(x)$ decays to 0 as $|x| \rightarrow \infty$ there exists a value $\lambda^{*} \geq 1$ such that $|x| \geq \lambda^{*}$ implies $v(x) \leq c_{1} / 2$. Let now $\lambda>\lambda^{*}$. Consider $x \in H_{\lambda}$ with $|x|>R(1)$. For such $x$ we have $|x|>R(\lambda)$ and hence $v(x)>v\left(x^{\lambda}\right)$ by Lemma 7 . Now consider $x \in H_{\lambda}$ with $|x| \leq R(1)$. Since $\left|x^{\lambda}\right| \geq \lambda>\lambda^{*}$ we find $v(x) \geq c_{1}>v\left(x^{\lambda}\right)$, and the claim is proved.

Case 2: $\alpha=N$. The proof is as above, but now $c_{1}$ is not necessarily positive. But now $v(x)$ decays to $-\infty$ as $|x| \rightarrow \infty$ so that we can choose the value $\lambda^{*} \geq 1$ such that $|x| \geq \lambda^{*}$ implies $v(x) \leq c_{1}-1$. The rest of the proof is the same.

Case 3: $\alpha>N$. Choose $c_{1}:=\max _{|x| \leq R(1)} v(x)$ so that $c_{1}>0$. This time $v(x)$ tends to $\infty$ as $|x| \rightarrow \infty$ so that we can choose $\lambda^{*} \geq 1$ such that $|x| \geq \lambda^{*}$ implies $v(x) \geq 2 c_{1}$. Similar consideration as before imply the claim.

Lemma 9. Let $\lambda>0$.

(a) For all $x, y \in H_{\lambda}$ :

$$
\begin{aligned}
2<\alpha<N: & \frac{1}{|x-y|^{N-\alpha}}>\frac{1}{\left|x^{\lambda}-y\right|^{N-\alpha}}, \\
\alpha=N: & \log \frac{1}{|x-y|}>\log \frac{1}{\left|x^{\lambda}-y\right|}, \\
\alpha>N: & \frac{1}{|x-y|^{N-\alpha}}<\frac{1}{\left|x^{\lambda}-y\right|^{N-\alpha}} .
\end{aligned}
$$

(b) For all $x \in T_{\lambda}, y \in H_{\lambda}$ :

$$
\begin{array}{rlrlrl}
2<\alpha<N: & \frac{\partial}{\partial x_{1}} \frac{1}{|x-y|^{N-\alpha}}<0, & \frac{\partial}{\partial x_{1}}\left(\frac{1}{|x-y|^{N-\alpha}}+\frac{1}{\left|x-y^{\lambda}\right|^{N-\alpha}}\right) & =0, \\
\alpha & =N: & \frac{\partial}{\partial x_{1}} \log \frac{1}{|x-y|}<0, & \frac{\partial}{\partial x_{1}}\left(\log \frac{1}{|x-y|}+\log \frac{1}{\left|x-y^{\lambda}\right|}\right) & =0, \\
\alpha>N: & \frac{\partial}{\partial x_{1}} \frac{1}{|x-y|^{N-\alpha}}>0, & \frac{\partial}{\partial x_{1}}\left(\frac{1}{|x-y|^{N-\alpha}}+\frac{1}{\left|x-y^{\lambda}\right|^{N-\alpha}}\right) & =0 .
\end{array}
$$

Proof. The proof of (a) follows from

$$
\left|x^{\lambda}-y\right|^{2}=4 \underbrace{\left(\lambda-x_{1}\right)}_{>0} \underbrace{\left(\lambda-y_{1}\right)}_{>0}+|x-y|^{2} .
$$

The proof of the first part of (b) follows from

$$
\frac{\partial}{\partial x_{1}}|x-y|=\frac{x_{1}-y_{1}}{|x-y|}=\frac{\lambda-y_{1}}{|x-y|}>0
$$

and the chain rule. For the second part of (b) note that if $x \in T_{\lambda}$ and $y \in H_{\lambda}$ then $|x-y|=\left|x^{\lambda}-y^{\lambda}\right|=\left|x-y^{\lambda}\right|$. Hence for every $C^{1}$-function $g$ we have that

$$
\frac{\partial}{\partial x_{1}}\left(g(|x-y|)+g\left(\left|x-y^{\lambda}\right|\right)\right)=\frac{g^{\prime}(|x-y|)}{|x-y|}\left(\left(x_{1}-y_{1}\right)+x_{1}-\left(2 \lambda-y_{1}\right)\right)=0
$$

since $x \in T_{\lambda}$.

Lemma 10. Let $\lambda>0$.

(a) Suppose $2<\alpha \leq N$. If $w_{\lambda} \geq 0$ in $H_{\lambda}$ then $w_{\lambda}>0$ in $H_{\lambda}$ and $\frac{\partial w_{\lambda}}{\partial x_{1}}(x)<0$ on $T_{\lambda}$.

(b) Suppose $\alpha>N$. If $w_{\lambda} \leq 0$ in $H_{\lambda}$ then $w_{\lambda}<0$ in $H_{\lambda}$ and $\frac{\partial w_{\lambda}}{\partial x_{1}}(x)>0$ on $T_{\lambda}$. 
Proof. We give the proof in the case $2<\alpha<N$; the proof in the case $\alpha \geq N$ is very similar. Note first that by Corollary 5 we have

$$
\begin{aligned}
v(x) & =\int_{\mathbb{R}^{N}} \frac{f_{H}(v(y)-\beta)}{|x-y|^{N-\alpha}} d y=\int_{H_{\lambda}} \ldots d y+\int_{\mathbb{R}^{N} \backslash H_{\lambda}} \ldots d y \\
& =\int_{H_{\lambda}} \frac{f_{H}(v(y)-\beta)}{|x-y|^{N-\alpha}}+\frac{f_{H}\left(v\left(y^{\lambda}\right)-\beta\right)}{\left|x-y^{\lambda}\right|^{N-\alpha}} d y .
\end{aligned}
$$

Therefore

$$
\begin{aligned}
v(x)-v\left(x^{\lambda}\right)= & \int_{H_{\lambda}} f_{H}(v(y)-\beta)\left(\frac{1}{|x-y|^{N-\alpha}}-\frac{1}{\left|x^{\lambda}-y\right|^{N-\alpha}}\right) d y \\
& +\int_{H_{\lambda}} f_{H}\left(v\left(y^{\lambda}\right)-\beta\right)\left(\frac{1}{\left|x-y^{\lambda}\right|^{N-\alpha}}-\frac{1}{\left|x^{\lambda}-y^{\lambda}\right|^{N-\alpha}}\right) d y \\
= & \int_{H_{\lambda}}\left(f_{H}(v(y)-\beta)-f_{H}\left(v\left(y^{\lambda}\right)-\beta\right)\right) \underbrace{\left(\frac{1}{|x-y|^{N-\alpha}}-\frac{1}{\left|x^{\lambda}-y\right|^{N-\alpha}}\right)}_{>0 \text { by Lemma } 9(\mathrm{a})} d y .
\end{aligned}
$$

Moreover, $\left.f_{H}(v(y)-\beta)-f_{H}\left(v\left(y^{\lambda}\right)-\beta\right)\right) \geq 0$ since $f_{H}$ is non-decreasing and $w_{\lambda} \geq 0$ by assumption. If we assume for contradiction that $f_{H}(v(y)-\beta) \equiv f_{H}\left(v\left(y^{\lambda}\right)-\beta\right)$ for almost all $y \in H_{\lambda}$ then we would find $v(x)-v\left(x^{\lambda}\right) \equiv 0$ in $H_{\lambda}$, which contradicts Lemma 7 and the assumption $\lambda>0$. Therefore there exists a subset $M_{\lambda} \subset H_{\lambda}$ of positive measure such that $f_{H}(v(y)-\beta)>f_{H}\left(v\left(y^{\lambda}\right)-\beta\right)$ for all $y \in M_{\lambda}$. As a consequence we see from (5) that $w_{\lambda}(x)>0$ for all $x \in H_{\lambda}$.

To see the second part of the claim, note that for $x \in T_{\lambda}$ we have $\frac{\partial w_{\lambda}}{\partial x_{1}}(x)=2 \frac{\partial v}{\partial x_{1}}(x)$ so that

$$
\begin{aligned}
& \frac{1}{2} \frac{\partial w_{\lambda}}{\partial x_{1}}(x) \\
& \quad=\int_{H_{\lambda}} f_{H}(v(y)-\beta) \underbrace{\frac{\partial}{\partial x_{1}}\left(\frac{1}{|x-y|^{N-\alpha}}\right)}_{<0 \text { by Lemma } 9(\mathrm{~b})}+f_{H}\left(v\left(y^{\lambda}\right)-\beta\right) \frac{\partial}{\partial x_{1}}\left(\frac{1}{\left|x-y^{\lambda}\right|^{N-\alpha}}\right) d y
\end{aligned}
$$

Moreover, we have seen that $f_{H}(v(y)-\beta)>f_{H}\left(v\left(y^{\lambda}\right)-\beta\right)$ on a subset $M_{\lambda} \subset H_{\lambda}$ of positive measure. Therefore, for all $x \in T_{\lambda}$ we find

$$
\frac{1}{2} \frac{\partial w_{\lambda}}{\partial x_{1}}(x)<\int_{H_{\lambda}} f_{H}\left(v\left(y^{\lambda}\right)-\beta\right) \frac{\partial}{\partial x_{1}}\left(\frac{1}{|x-y|^{N-\alpha}}+\frac{1}{\left|x-y^{\lambda}\right|^{N-\alpha}}\right) d y=0
$$

due to Lemma 9(b). This establishes the claim.

For the final part of this section let us define the set

$$
J:= \begin{cases}\left\{\lambda>0: w_{\lambda}>0 \text { in } H_{\lambda}\right\} & \text { if } 2<\alpha \leq N \\ \left\{\lambda>0: w_{\lambda}<0 \text { in } H_{\lambda}\right\} & \text { if } \alpha>N .\end{cases}
$$

Lemma 11. The set $J \subset(0, \infty)$ is open.

Proof. We give the proof only in the case $2<\alpha \leq N$. Assume that $J$ is not open. Then for some $\lambda \in J$ there exists a sequence $\lambda_{n} \rightarrow \lambda$ as $n \rightarrow \infty$ and $x_{n} \in H_{\lambda_{n}}$ such that $w_{\lambda_{n}}\left(x_{n}\right) \leq 0$. Let $R(\lambda)$ be the function from Lemma 7 . Clearly $\left|x_{n}\right| \leq R(\lambda / 2)$, because $\left|x_{n}\right|>R(\lambda / 2)$ would imply $\left|x_{n}\right|>R\left(\lambda_{n}\right)$ for large $n$ and hence $w_{\lambda_{n}}\left(x_{n}\right)>0$ for large $n$, which cannot hold. Hence, by extracting a subsequence if necessary, we may assume that 
$x_{n} \rightarrow x_{0} \in \overline{B_{R(\lambda / 2)}(0)}, x_{0} \in \overline{H(\lambda)}$. Since $w_{\lambda}>0$ in $H_{\lambda}$ we must have $x_{0} \in T_{\lambda}$. Thus, by Lemma $10\left(\right.$ a) we find $\frac{\partial v}{\partial x_{1}}\left(x_{0}\right)<0$, which contradicts $v\left(x_{n}\right) \leq v\left(x_{n}^{\lambda_{n}}\right)$ for large $n$.

The proof of Theorem 2 will be completed through the following, final lemma.

Lemma 12. The set $J=(0, \infty)$.

Proof. Again let us stay with the case $2<\alpha \leq N$. Let $(\mu, \infty)$ be the largest open interval contained in $J$. By Lemma $8, \mu$ is a finite value in $[0, \infty)$. Assume for contradiction that $\mu>0$. Then $w_{\mu} \geq 0$ in $H_{\mu}$ and by Lemma 10(a) we see that $w_{\mu}>0$ in $H_{\mu}$ so that $\mu \in J$. A contradiction is reached since by Lemma 11 we know that $J$ is open.

\section{REFERENCES}

[1] A.D. Alexandrov, A characteristic property of the spheres. Ann. Mat. Pura Appl. 58 (1962), 303354.

[2] E. Berchio, F. Gazzola and T. Weth, Radial symmetry of positive solutions to nonlinear polyharmonic Dirichlet problems. To appear in J. Reine Angew. Math.

[3] M. Birkner, J.A. López-Mimbela, A. Wakolbinger, Comparison results and steady states for the Fujita equation with fractional Laplacian. Ann. Inst. H. Poincaré Anal. Non Linéaire 22 (2005), 83-97.

[4] Sun-Yung A. Chang, Paul C. Yang, On uniqueness of solutions of nth order differential equations in conformal geometry. Math. Res. Lett. 4 (1997), 91-102.

[5] Wenxiong Chen, Congming Li and Biao Ou, Classification of solutions for an integral equation. Comm. Pure Appl. Math. 59 (2006), 330-343.

[6] P. Ebenfelt, D. Khavinson and H.S. Shapiro, A free boundary problem related to single-layer potentials. Ann. Acad. Sci. Fenn. Math. 27 (2002), 21-46.

[7] L.E. Fraenkel, Introduction to maximum principles and symmetry in elliptic problems, Cambridge tracts in mathematics 128, Cambridge University Press 2000.

[8] S.J. Gardiner, An equilibrium measure characterization of circles. Forum Math. 14 (2002), 953-954.

[9] B. Gidas, Wei-Ming Ni and L. Nirenberg, Symmetry and related properties via the maximum principle. Comm. Math. Phys. 68 (1979), 209-243.

[10] E. Heil and H. Martini, Special convex bodies. In Handbook of Convex Geometry, Ed. P. Gruber \& J. Wills, Elsevier (1993), 347-385.

[11] Yan Yan Li: Remark on some conformally invariant integral equations, the method of moving spheres. J. Eur. Math. Soc. 6 (2004), 153-180.

[12] Mikyoung Lim, Symmetry of a boundary integral operator and a characterization of a ball. Illinois J. Math. 45 (2001), 537-543.

[13] E. Martensen, Eine Integralgleichung für die logarithmische Gleichgewichtsbelegung und die Krümmung der Randkurve eines ebenen Gebiets. Z. Angew. Math. Mech. 72 (1992), T596-T599.

[14] O. Mendez and W. Reichel, Electrostatic characterization of spheres. Forum Math. 12 (2000), 223245.

[15] L. Payne and G. Philippin, On some maximum principles involving harmonic functions and their derivatives. SIAM J. Math. Anal. 10 (1979), 96-104.

[16] G. Philippin, On a free boundary problem in electrostatics. Math. Meth. Appl. Sci. 12 (1990), 387-392.

[17] W. Reichel, Radial symmetry for elliptic boundary value problems on exterior domains. Arch. Rational Mech. Anal. 137 (1997), 381-394.

[18] J. Serrin, A symmetry problem in potential theory. Arch. Ration. Mech. Anal. 43 (1971), 304-318.

[19] B. Sirakov, Symmetry for exterior elliptic problems and two conjectures in potential theory. Ann. Inst. H. Poincaré Anal. Non Linéaire 18 (2001), 135-156.

[20] H. Shahgholian, A characterization of the sphere in terms of single-layer potentials. Proc. Amer. Math. Soc. 115 (1992), 1167-1168.

WOLFGANG REICHEL

Institut FÜR ANALYSis, Universität KARLSRUhe

Englerstr. 2, D-76128 Karlsruhe, Germany 